\title{
Fiebre manchada de montañas rocosas: experiencia en 5 años de vigilancia activa en un hospital pediátrico de segundo nivel en el noreste de México
}

\author{
Delmy C. López-Castillo ${ }^{1}$, Denisse Vaquera-Aparicio ${ }^{1}$, Miguel A. González-Soto ${ }^{1}$, \\ Rogelio Martínez-Ramírez ${ }^{1}$, Lorena Rodríguez-Muñoz ${ }^{1 *}$ y Fortino Solórzano-Santos ${ }^{2}$ \\ ${ }^{1}$ Hospital del Niño, Secretaria de Salud, Saltillo, Coah; ${ }^{2}$ Unidad de Investigación Medicina Basada en Evidencias, Hospital Infantil de México \\ Federico Gómez, Ciudad de México. México
}

\begin{abstract}
Resumen
Introducción: La fiebre manchada de las montañas rocosas (FMMR) es causada por Rickettsia rickettsii. En Coahuila, la región de la Comarca Lagunera se considera una zona endémica. No se han reportado casos en la zona sur del estado, específicamente en la ciudad de Saltillo. Métodos: Estudio prospectivo, reporte de casos. Se incluyeron los casos de niños atendidos en el Hospital del Niño Dr. Federico Gómez Santos en la ciudad de Saltillo, Coah., del mes de septiembre de 2012 a septiembre 2017 con diagnóstico confirmado clínicamente y por reacción en cadena de la polimerasa (PCR) de FMMR. Se presentan los antecedentes epidemiológicos, las características clínicas y evolución de los pacientes. Resultados: Se confirmaron 14 pacientes. La relación masculino:femenino fue de 1.8:1, la edad promedio de los pacientes fue de 7.6 años (18 meses a 13 años). El $42.8 \%$ de los pacientes refirió el contacto con perros y el $57.1 \%$ afirmó tener contacto con garrapatas. En todos los casos hubo fiebre y exantema purpúrico; alrededor del $70 \%$ manifestaron mialgias y artralgias; el $28 \%$ tuvo sangrado del tubo digestivo, y el $11 \%$ alteraciones neurológicas graves. El $64.2 \%$ de los casos recibió tratamiento adecuado con doxiciclina. Fallecieron 8 pacientes, con una tasa de letalidad de 57.1\%. Conclusiones: La zona sur de Coahuila debe considerarse una zona endémica para FMMR. El retraso en el diagnóstico y tratamiento favorecen una mayor letalidad.
\end{abstract}

Palabras clave: Fiebre manchada de montañas rocosas. Rickettsia rickettsii. Exantema purpúrico. Garrapatas.

\section{Rocky mountain spotted fever: five years of active surveillance experience in a second level pediatric hospital in northeastern Mexico}

\section{Abstract}

Background: Rocky Mountain Spotted Fever (RMSF) is caused by Rickettsia rickettsii. In Coahuila, Comarca Lagunera is considered an endemic zone; no cases have been reported in the southern zone of the state, specifically in the city of Saltillo.

\section{Correspondencia:}

*Lorena Rodríguez-Muñoz

E-mail: dra.lorenardzm@gmail.com
Disponible en internet: 25-09-2018 Bol Med Hosp Infant Mex. 2018;75:303-308 www.bmhim.com

1665-1146/@ 2018. Hospital Infantil de México Federico Gómez, impreso por Permanyer México SA de CV, todos los derechos reservados. 
Methods: Prospective study, cases report. Children evaluated in the Hospital del Niño Dr. Federico Gómez Santos from September 2012 to September 2017, with clinically and laboratory (by polymerase chain reaction, PCR) confirmed diagnosis of FMMR were included. The epidemiological antecedents, clinical characteristics and patient's evolution are presented. Results: 14 patients were confirmed. The male: female ratio was 1.8: 1, the average age of the patients was 7.6 years (18 months to 13 years). $42.8 \%$ reported contact with dogs and $57.1 \%$ confirmed contact with ticks. In all cases, there was fever and purpuric rash; around $70 \%$ manifested myalgias and arthralgias; $28 \%$ presented digestive tract bleeding and $11 \%$ had severe neurological alterations. $64.2 \%$ of the cases received adequate treatment with doxycycline. Eight patients died with a case fatality rate of $57.1 \%$. Conclusions: The southern zone of Coahuila should be considered an endemic area for FMMR. The delay in diagnosis and treatment favor a greater lethality.

Key words: Rocky mountain spotted fever. Rickettsia rickettsii. Purpuric exanthema. Ticks.

\section{Introducción}

Las enfermedades causadas por rickettsias son zoonosis que afectan en la naturaleza a pequeños mamíferos y vectores. Los humanos son huéspedes accidentales y son infectados principalmente por la garrapata de animales silvestres y peridomesticos ${ }^{1}$. Las rickettsiosis en humanos frecuentemente comparten características clínicas, a pesar de ser etiológicamente y epidemiológicamente distintas. En EE.UU., estas enfermedades incluyen fiebre manchada de las montañas rocallosas (FMMR), causada por Rickettsia rickettsii, y un grupo de otras fiebres manchadas (SFG), causadas por Rickettsia pakeri y Rickettsia especie 364D. Las rickettsiosis transmitidas por artrópodos diferentes a garrapatas incluyen Rickettsia typhi, transmitida por pulgas, Rickettsia prowazekiii, transmitida por piojos, y Rickettsia akari, transmitida por ácaros ${ }^{2}$.

A pesar de que en México se han descrito varias especies patógenas para los humanos, $R$. rickettsii es la de mayor importancia ${ }^{3}$.

En los últimos 100 años, los casos fatales durante los brotes de fiebre manchada en México van del 30 al $80 \%$, una mortalidad que excede incluso otras infecciones fatales como meningococcemia, fiebre amarilla y plaga ${ }^{4}$.

$R$. rickettsii es una bacteria intracelular obligada, cocobacilo pleomórfico gramnegativo, no móvil, de aproximadamente $0.3 \mu \mathrm{m}$ a $1.00 \mu \mathrm{m}$. No puede cultivarse en medios convencionales, sino que requiere cultivos celulares especiales ${ }^{5}$.

Las enfermedades transmitidas por garrapatas ocurren en diversas áreas de Norteamérica, aunque no todas las enfermedades se presentan en todas las regiones. La FMMR fue descrita inicialmente en el norte de las Montañas Rocosas; sin embargo, los casos se agrupan principalmente en el sureste y regiones del medio Atlántico de EE.UU. La FMMR se ha reportado en todo EE.UU., exceptuando Alaska y Hawái; asimismo se presenta en México, Centro y América del Sur. En Brasil incluso se conoce como fiebre manchada brasileña ${ }^{6-9}$.

Las rickettsiosis fueron reconocidas en México desde mediados de 1930, principalmente en los estados fronterizos del norte, con persistencia de diversos brotes en los estados de Sinaloa, Sonora, Durango y Coahuila. En 1945, Bustamante, Ortiz Mariotte y Varela identificaron como vector a la garrapata del perro, Rhipicephalus sanguineus, en Sinaloa ${ }^{3}$. Diversos investigadores han demostrado también su presencia en todo el norte de México, y han encontrado además que los perros de las comunidades afectadas están infestados con este vector. También se ha detectado en las paredes de adobe, pisos de tierra y colchones de los hogares afectados, lo que explica la mayor ocurrencia de casos en niños y mujeres, perfil epidemiológico que difiere en México de lo descrito en EE.UU. ${ }^{4}$.

En una etapa de varias décadas no hubo información, pero a finales del siglo $X X$ y en los inicios del siglo $X X I$ se presentó una reemergencia.

En el año 2009 se emitió una alerta epidemiológica por brote de rickettsiosis en Baja California, con un total de 1,471 casos sospechosos en todo el año. El 24 de abril de 2015, el Centro Nacional de Programas Preventivos y Control de Enfermedades (CENAPRECE) del Ministerio de Salud de México emitió una Declaración de Emergencia Epidemiológica para la FMMR en las regiones del norte de México, particularmente en los estados de Baja California y Sonora ${ }^{4}$. La comarca lagunera representa un foco persistente de FMMR en México. En un hospital pediátrico de Torreón, Coahuila, se estudiaron 115 casos tratados del 1975 al 2007, y se describieron características clínicas y epidemiológicas muy similares a las de los brotes reportados en las décadas de 1940 y $1950^{4}$.

La incidencia de rickettsiosis en México es baja: la Dirección General de Epidemiología tiene reporte del año 2000 al 2008 de solo 0 a 9 casos por de 


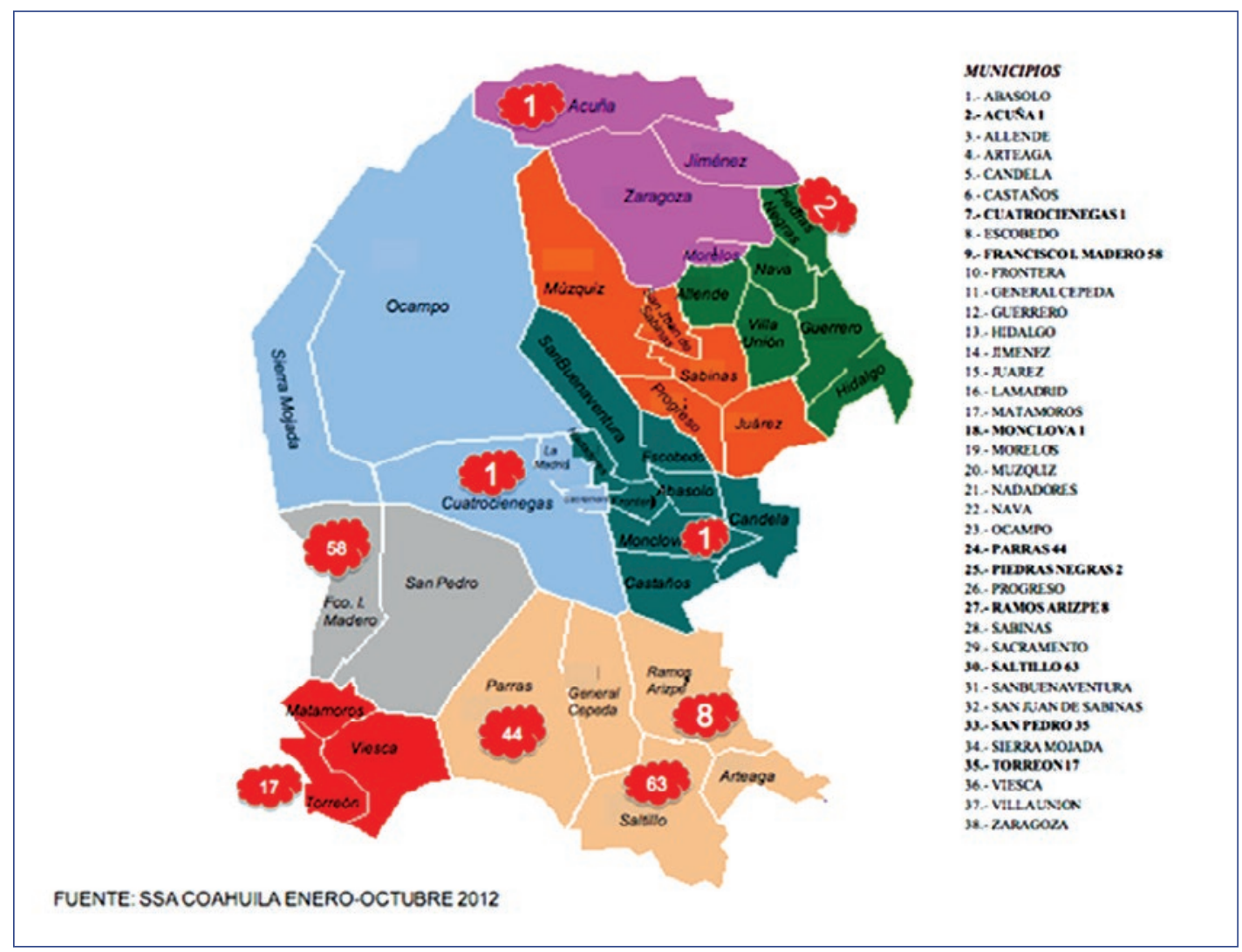

Figura 1. Municipios del estado de Coahuila que presentaron casos sospechosos de rickettsiosis en el año 2012.

rickettsiosis (fiebre manchada, tifo murino y tifo epidémico). A partir del año 2009 existe un incremento en el número de casos notificados, y el promedio es de 500 casos por año de fiebre manchada, con una inci-

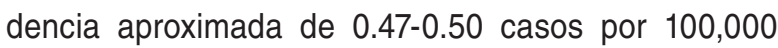
habitantes. Respecto a los padecimientos de tifo murino y epidémico, la incidencia se reporta mucho más baja, con aproximadamente 0.09 casos por 100,000 habitantes al año $0^{10,11}$.

En el Hospital del Niño Dr. Federico Gómez Santos de Saltillo, Coahuila, se detectó por primera vez un brote de casos de fiebre manchada en septiembre del 2012, y fue el primer caso reportado en la ciudad. Históricamente, los casos de fiebre manchada en el estado de Coahuila eran reportados en la comarca lagunera, pero no en la región sur del estado ${ }^{3}$ (Fig. 1). A raíz de los casos documentados, se dio un aviso epidemiológico de brote por parte de la Dirección General de Epidemiología para poner énfasis en el diagnóstico y detección oportuna de casos. Desde esa fecha y hasta la actualidad se siguen presentando casos de fiebre manchada por $R$. rickettsii de forma endémica en esta región.

En este estudio se presenta una serie de casos detectados en esta región del noreste del país.

\section{Métodos}

A partir de septiembre de 2012 y hasta el mes de septiembre de 2017, se colectó en forma prospectiva la información de pacientes atendidos en el Hospital del Niño, en quienes se confirmó el diagnóstico de FMMR. En todos los casos, la confirmación diagnóstica se realizó mediante la prueba de reacción en cadena de la polimerasa (PCR) para el gen gltA9, y se reportó como positivo un valor de Ct (umbral de ciclo, por sus siglas en inglés, cycle threshold) de 20 en el suero de Ios pacientes. La prueba de PCR fue realizada en el Instituto Nacional de Referencia Epidemiológica de la Secretaria de Salud, México. No se realizaron pruebas serológicas debido al momento en que fueron captados los pacientes, entendiendo que de 0 a 14 días del 
inicio de los síntomas la prueba diagnóstica debe hacerse por PCR y del quinceavo día en adelante se realizan pruebas de serología; esto con base en la actualización de lineamientos de Rickettsiosis en el Manual de Vigilancia Epidemiológica de Enfermedades Transmitidas por Vector ${ }^{12}$.

Se analizaron características epidemiológicas generales, contacto con garrapatas, contacto con mascotas, manifestaciones clínicas, principales hallazgos de laboratorio y respuesta al tratamiento. Los resultados se presentan en forma descriptiva.

El estudio fue autorizado por el Comité de Ética en Investigación del Hospital del Niño. Por las características del estudio no se solicitó carta de autorización a familiares.

\section{Resultados}

En el periodo descrito se confirmaron 14 pacientes. La relación masculino:femenino fue de 1.8:1, la edad promedio de los pacientes fue de 7.6 años (18 meses a 13 años). El $42.8 \%$ de los pacientes refirió el contacto con perros y $57.1 \%$ confirmó el contacto con garrapatas. La frecuencia de signos y síntomas de los pacientes, así como las alteraciones más importantes de laboratorio se muestran en la tabla 1. En todos los casos se presentó fiebre y exantema purpúrico; alrededor del $70 \%$ de los pacientes manifestaron mialgias y artralgias. Como manifestaciones de gravedad, el $28 \%$ tuvo sangrado del tubo digestivo y el $11 \%$, alteraciones neurológicas graves.

A siete de los pacientes se les tomó reacción de Weil-Felix con una mediana de títulos de 1:80; cinco casos tuvieron títulos iguales o mayores a 1:80. El $64.2 \%$ de los casos recibió tratamiento adecuado con doxiciclina (a $4.4 \mathrm{mg} / \mathrm{kg} /$ día por lo menos 7 días); dos pacientes recibieron el antibiótico intravenoso. La mediana de días de retraso para el inicio de antibiótico fue de 3.6 días, con un rango de 0 a 8 días en los sobrevivientes y de 4.3 días en los que fallecieron; los dos pacientes que recibieron doxiciclina intravenosa sobrevivieron. Fallecieron ocho pacientes, con una tasa de letalidad de $57.1 \%$; las principales causas de muerte fueron choque, sangrado del tubo digestivo e insuficiencia respiratoria grave. Ninguno de los sobrevivientes presentó alguna secuela.

En un paciente se administró inmunoglobulina intravenosa (IGIV), y en dos pacientes, bolos de metilprednisolona, los cuales tuvieron una mejor evolución; sin embargo, dadas las características del estudio, no se
Tabla 1. Principales signos, síntomas y alteraciones de laboratorio en 14 niños con fiebre manchada de Montañas Rocosas

\begin{tabular}{|l|c|}
\hline Signos, síntomas, alteraciones de laboratorio & Frecuencia \\
\hline Fiebre & $100 \%$ \\
\hline Exantema & $100 \%$ \\
\hline Cefalea & $78.5 \%$ \\
\hline Dolor abdominal & $78.5 \%$ \\
\hline Mialgias y artralgias & $71.4 \%$ \\
\hline Náuseas o vómitos & $71.4 \%$ \\
\hline Irritabilidad & $50 \%$ \\
\hline Diarrea & $50 \%$ \\
\hline Hiporexia & $35.7 \%$ \\
\hline Datos de sangrado (sangrado de tubo digestivo, & $28.5 \%$ \\
\hline petequias, epistaxis) & \\
\hline Convulsiones o déficit neurológico focalizado & $11.4 \%$ \\
\hline Plaquetopenia & $92.3 \%$ \\
\hline Hiponatremia & $92.3 \%$ \\
\hline Elevación de enzimas hepáticas & $92.3 \%$ \\
\hline Tiempos de coagulación prolongados & $84.6 \%$ \\
\hline
\end{tabular}

puede establecer el efecto benéfico de estos productos.

\section{Discusión}

La FMMR es una enfermedad causada por la bacteria $R$. rickettsii, que es transmitida por garrapatas, ampliamente diseminadas en la zona norte de nuestro país ${ }^{4}$.Desafortunadamente, aun en la actualidad, tiene una alta letalidad por su rápida evolución y, en lugares no endémicos, por la falta de sospecha en esta enfermedad, como queda ejemplificado con los casos reportados en la frontera México-EE.UU. ${ }^{13}$. El inicio de la infección se caracteriza por signos y síntomas inespecíficos como la fiebre, el dolor de cabeza y el dolor muscular, que suelen presentarse en una amplia gama de enfermedades infecciosas. Dada la baja frecuencia de esta enfermedad, en muchos de los estados del país es probable que no se sospeche la enfermedad hasta momentos muy tardíos. Un signo orientador suele ser el exantema, que va de maculopapular a purpúrico. Esta última forma clínica suele ser la más orientadora; como pudo observarse en esta serie, el $100 \%$ de los casos tuvo exantema, lo cual es 
semejante a lo informado en otros estudios en Méxi$\mathrm{CO}^{14}$. Es conveniente insistir que, en zonas endémicas del país, en los niños con fiebre y exantema (especialmente si es de características purpúricas), debe incluirse en el diagnóstico diferencial infección por rickettsias.

El retraso en el diagnóstico clínico condiciona que el daño fisiopatogénico causado por $R$. rickettsii se generalice. Se conoce que existe daño de las células endoteliales de los vasos sanguíneos. Esta vasculitis genera daño tisular multisistémico, que progresivamente provoca insuficiencia orgánica, necrosis cutánea y muerte. Se ha considerado que la plaquetopenia, la alteración en las pruebas de coagulación y la elevación de enzimas hepáticas son parámetros de laboratorio de mal pronóstico que se detectaron en el alrededor del $90 \%$ de los niños en esta serie.

La mayoría de las muertes relacionadas con FMMR ocurren dentro de la primera y segunda semanas de evolución; generalmente se deben a choque, complicaciones pulmonares, sobrecarga circulatoria y daño miocárdico o renal. La combinación de varios factores parece influir en la letalidad, principalmente la falta de sospecha diagnóstica que conlleva a retraso en el inicio del tratamiento indicado, a lo cual se suma la virulencia de $R$. rickettsii y en algunos casos, el mal manejo de líquidos ${ }^{14-16}$.

A pesar de haber mantenido una alerta epidemiológica para la detección oportuna de casos en nuestro hospital, la letalidad fue muy elevada, probablemente por el momento tardío en que los pacientes acudieron o fueron enviados. En las diversas series que analizan letalidad, la mayoría de los pacientes han sido vistos previamente al menos por dos médicos ${ }^{3,13,14}$. Es de resaltar que, de los pacientes en este estudio, aquellos que iniciaron tratamiento después del quinto día son los que tuvieron mayor letalidad. Recientemente, Álvarez-Hernández et al. identificaron que en un estado endémico del país, solo el $62 \%$ de los médicos estuvo de acuerdo en el uso de doxiciclina en menores de 8 años de edad, solo el $40 \%$ de los médicos de primer contacto identificó el momento oportuno del inicio de tratamiento y solo el $32 \%$ asocia el inicio tardío de tratamiento como un factor influyente en la mayor letalidad ${ }^{17}$.

En algunas unidades médicas se continúa utilizando la prueba de Weil-Felix dentro de las pruebas iniciales de estudio de los pacientes. En zonas endémicas, esta prueba puede considerarse como positiva a un valor de corte $\geq 1: 160$ y en zonas no endémicas, $\geq 1: 80$. Sin embargo, la sensibilidad y especificidad de la prueba
(Weil-Felix) es baja para el diagnóstico de la FMMR. Debe darse valor a una prueba positiva con datos clínicos sugestivos; una prueba negativa no descarta la enfermedad en zonas endémicas si la prueba se hace en etapas tempranas de la enfermedad. Aunque se puede utilizar esta prueba como elemento inicial de la valoración de los pacientes, se recomienda siempre pasar a otras pruebas como inmunofluorescencia, ELISA (por sus siglas en inglés Enzyme-linked Immunosorbent Assay) o PCR en los casos de alta sospecha clínica. A pesar de su alta especificidad, la prueba de PCR puede no detectar los casos cuando la prueba se hace en etapas tempranas de la enfermedad ${ }^{18,19}$. Este último punto nos permite considerar que, de los pacientes atendidos en el período de estudio, el número de casos pudo haber sido subestimado debido a que solo se incluyeron pacientes confirmados por PCR.

Existe consenso en cuanto a que el inicio temprano de doxiciclina mejora el pronóstico de los pacientes, por lo que, ante la sospecha clínica, debe iniciarse el tratamiento, aun en menores de 8 años de edad ${ }^{2}$. En países donde son frecuentes otras enfermedades infecciosas, como meningococcemias o infecciones graves por Streptococcus pneumoniae, deberá añadirse ceftriaxona.

En el presente estudio, a dos pacientes se les administró metilprednisolona, y en uno, además, se le indicó manejo con gammaglobulina intravenosa, considerando la fisiopatogenia de la enfermedad (vasculitis) y la información publicada de un paciente con FMMR, quien posteriormente presentó enfermedad de Kawasaki y mejoró con la administración de gammaglobulina intravenosa ${ }^{20}$. Estos dos pacientes sobrevivieron; sin embargo, no hay estudios sólidos que avalen esta indicación.

Un reto de salud pública muy importante en el país es que esta enfermedad es transmitida por una garrapata (Rhipicephalus sanguineus sensu lato) que infesta a los perros, por lo que la vivienda en áreas con bajo nivel socioeconómico donde existe gran número de perros callejeros y el contacto estrecho con perros domesticados con mal control de ectoparásitos favorece la persistencia de la enfermedad en zonas endémicas y su diseminación a otras zonas geográficas.

Con base en los datos presentados la zona sur del estado de Coahuila, esta ciudad debe considerarse un área endémica para la FMMR. Se ha registrado hasta ahora una alta tasa de letalidad en nuestro medio por el establecimiento de un diagnóstico en forma tardía, por lo que se recomienda que, en zonas endémicas, esta entidad debe ser sospechada en todo paciente 
con fiebre y exantema que reconozca haber tenido contacto con perros o garrapatas.

\section{Conflicto de intereses}

Los autores declaran no tener ningún conflicto de intereses.

\section{Responsabilidades éticas}

Protección de personas y animales. Los autores declaran que para esta investigación no se han realizado experimentos en seres humanos ni en animales.

Confidencialidad de los datos. Los autores declaran que han seguido los protocolos de su centro de trabajo sobre la publicación de datos de pacientes.

Derecho a la privacidad y consentimiento informado. Los autores declaran que en este artículo no aparecen datos de pacientes.

\section{Bibliografía}

1. Drexler N, Miller M, Gerding J, Todd S, Adams L, Dahlgren FS, et al Community-based control of the brown dog tick in a region with high rates of Rocky Mountain spotted fever, 2012-2013. PLoS ONE. 2014:9:1-18.

2. Biggs HM, Behravesh CB, Bradley KK, Dahlgren FS, Drexler NA Dumler JS, et al. Diagnosis and management of tickborne Rickettsial Diseases: Rocky Mountain spotted fever and other spotted fever group Rickettsioses, Ehrlichioses, and Anaplasmosis - United States. MMWR Recomm Rep. 2016;65:1-44

3. De Lara Huerta J, Cárdenas Barragán R. Fiebre manchada de las Montañas Rocosas en pediatría. Revisión clínica de una serie de 115 casos. Rev Enf Infec Pediatr. 2008;22:4-9.

4. Álvarez-Hernández G, Roldán JFG, Milan NSH, Lash RR, Behravesh CB, Paddock CD. Rocky Mountain spotted fever in Mexico: past, present, and future. Lancet Infect Dis. 2017;17:e189-96.
5. Woods CR. Rocky Mountain spotted fever in children. Pediatr Clin N Am. 2013;60:455-70

6. Parola P, Paddock CD, Socolovschi C, Labruna MB, Mediannikov O, Kernif $\mathrm{T}$, et al. Update on tick-borne rickettsioses around the world: a geographic approach. Clin Microbiol Rev. 2013;26:657-702.

7. Paris DH, Dumler JS. State of the art of diagnosis of rickettsial diseases: the use of blood specimens for diagnosis of scrub thypus, spotted fever group rickettsiosis, and murine thypus. Curr Opin Infect Dis. 2016;29:433-9.

8. Mukkada S, Buckingham SC. Recognition of and prompt treatment for tick-borne infections in children. Infect Dis Clin N Am. 2015;29:539-55.

9. Zavala-Castro JE, Dzul-Rosado K, Arias León JJ, Walker DH, Zavala-Velázquez JE. An Increase in Human Cases of Spotted Fever Rickettsiosis in Yucatan, México, Involving Children. Am J Trop Med Hyg. 2008;79: 907-10.

10. Field-Cortazares J, Escárcega-Avila AM, López-Valencia, Barreras-Serrano A, Tinoco-Gracia L.Seroprevalence and risk factors associated with rickettsiosis (Rickettsia rickettsii) in humans in Baja California, Mexico. Gac Med Mex. 2015;151:38-42.

11. Sistema Único de Información para la Vigilancia Epidemiológica/Dirección General de Epidemiología/Secretaría de Salud. (Acceso 3 de noviembre 2017). http://www.gob.mx/salud/acciones-y-programas/direccion-general-de-epidemiologia-boletin-epidemiologico

12. Manual de Procedimientos Estandarizados para la Vigilancia Epidemiológica de las Enfermedades Transmitidas por Vectores. Dirección General de Epidemiología, 2015.

13. Drexler NA, Yaglom H, Casal M, Fierro M, Kriner P, Murphy B, et al. Fatal Rocky Mountain spotted fever along the United States-Mexico Border, 2013-2016. Emerg Infect Dis. 2017;23:1621-6.

14. Álvarez-Hernández G, Candia-Plata Mdel C, Delgado-de la Mora J, Acuña-Meléndrez NH, Vargas-Ortega AP, Licona-Enríquez JD. Rocky Mountain spotted fever in Mexican children: Clinical and mortality factors. Salud Públ Méx. 2016:58:385-92.

15. Martínez-Medina MÁ, Rascón-Alcantar A. Complications and cause of death in Mexican children with rocky mountain spotted fever. Gac Med Mex. 2016;152:789-95.

16. Regan JJ, Traeger MS, Humpherys D, Mahoney DL, Martinez M, Emerson GL, et al. Risk factors for fatal outcome from Rocky Mountain spotted fever in a highly endemic area- Arizona, 2002- 2011. Clin Infect Dis. 2015;60:1659-66

17. Alvarez-Hernandez G, Ernst K, Acuña-Melendrez NH, Vargas-Ortega AP, Candia-Plata MDC. Medical knowledge related to Rocky Mountain spotted fever in Sonora, Mexico. Trans R Soc Trop Med Hyg. 2018;112:109-14.

18. Levin ML, Snellgrove AN, Zemtsova GE. Comparative value of blood and skin samples for diagnosis of spotted fever group rickettsial infection in model animals. Ticks Tick Borne Dis. 2016;7:1029-34.

19. Tripathi CDP, Singh M, Agarwal J, Kanta C, Atam V. Seroepidemiology of Spotted Fever Rickettsiosis in Uttar Pradesh: A Prospective Study. J Clin Diagn Res. 2017;11:DC04-9.

20. Bal A, Kairys S. Kawasaki disease following Rocky Mountain spotted fever: a case report. J Med Case Reports. 2009;3:7320. 